\section{Guidelines of the American College of Obstetricians and Gynecologists for exercise during pregnancy and the postpartum period}

\section{R Artal, M O'Toole}

\section{New guidelines for exercise in pregnancy and postpartum have been published by the American College of Obstetricians and Gynecologists}

\begin{abstract}
n January 2002 the American College of Obstetricians and Gynecologists (ACOG) published new recommendations and guidelines for exercise during pregnancy and the postpartum period. Regular exercise is promoted for its overall health benefits. Pregnancy is recognised as a unique time for behaviour modification and is no longer considered a condition for confinement. It is currently recognised that habits adopted during pregnancy could affect a woman's health for the rest of her life. For the first time the recommendation suggests a possible role for exercise in the prevention and management of gestational diabetes.
\end{abstract}

The recommendations also promote exercise for sedentary women and those with medical or obstetric complications, but only after medical evaluation and clearance.

Box 1 lists the absolute contraindications to aerobic exercise during pregnancy, and box 2 the relative contraindications. As with any form of exercise prescription, these recommendation also include the warning signs to terminate exercise while pregnant (box 3 ). The recommendations also offer guidelines for sports and recreational activities. It cautions against participation in contact sports and recommends avoidance of scuba diving.

As for postpartum resumption of activities, the recommendations note that rapid resumption has no adverse effects, but gradual return to former activities is advised. This review includes background and comments to the above recommendations.

The health benefits of physical activity are well recognised, and conversely sedentary habits and low levels of cardiorespiratory fitness are leading risk factors for subsequent development of cardiovascular disease. ${ }^{2}$

The Centers for Disease Control and Prevention and the American College of

Sports Medicine (CDC-ACSM) have recommended the accumulation of $30 \mathrm{~min}$ utes or more of moderate intensity physical activity on most, and preferably all, days of the week. ${ }^{4}$ Moderate intensity physical activity is defined as activity with an energy requirement of 3-5 metabolic equivalents (METS). For most healthy adults, this is equivalent to brisk walking at 3-4 mph. The CDC-ACSM statement also recognises that more intense exercise performed in 20-60 minute sessions on three to five days a week will result in higher levels of physical fitness.

Despite the fact that pregnancy is associated with profound anatomical and physiological changes, there are few instances that should preclude otherwise healthy, pregnant women from following the same recommendations.

\section{MUSCULOSKELETAL \\ ADAPTATIONS}

Anatomical and physiological changes during pregnancy have the potential to affect the musculoskeletal system at rest and during exercise. The most obvious of these is weight gain. The increased weight in pregnancy may significantly increase the forces across joints such as the hips and knees by as much as $100 \%^{5}$ during weight bearing exercise such as running. Such large forces may cause discomfort to normal joints and increase damage to arthritic or previously unstable joints.

\begin{abstract}
"Despite a lack of clear evidence that musculoskeletal injuries are increased during pregnancy, these possibilities should nevertheless be considered when prescribing exercise in pregnancy."
\end{abstract}

Data on the effects of increased weight of pregnancy on joint injury and pathology are lacking. Because of anatomical changes, pregnant women typically develop lumbar lordosis, which contributes to the very high prevalence $(50 \%)$ of low back pain in pregnant women. Balance may be affected by changes in posture, predisposing pregnant women to loss of balance and increased risk of falling. However, increased incidence of falling during pregnancy has not been reported. Another musculoskeletal change during pregnancy is increased ligamentous laxity thought to be secondary to the influence of the increased levels of oestrogen and relaxin. Theoretically, this would predispose pregnant women to increased incidence of strains and sprains. This hypothesis has been substantiated by objective data on the metacarpophalangeal joints. ${ }^{6}$ Despite a lack of clear evidence that musculoskeletal injuries are increased during pregnancy, these possibilities should nevertheless be considered when prescribing exercise in pregnancy.

Box 1 Absolute contraindications to aerobic exercise during pregnancy (with permission from ACOG')

- Haemodynamically significant heart disease

- Restrictive lung disease

- Incompetent cervix/cerclage

- Multiple gestation at risk for premature labour

- Persistent second or third trimester bleeding

- Placenta praevia after 26 weeks gestation

- Premature labour during the current pregnancy

- Ruptured membranes

- Pregnancy induced hypertension

Box 2 Relative contraindications to aerobic exercise during pregnancy (with permission from ACOG')

- Severe anaemia

- Unevaluated maternal cardiac ar rhythmia

- Chronic bronchitis

- Poorly controlled type I diabetes

- Extreme morbid obesity

- Extreme underweight (body mass index $<12$ 1

- History of extremely sedentary lifestyle

- Intrauterine growth restriction in current pregnancy

- Poorly controlled hypertension/preeclampsia

- Orthopaedic limitations

- Poorly controlled seizure disorder

- Poorly controlled thyroid disease

- Heavy smoker 
Box 3 Warning signs to

terminate exercise while pregnant

- Vaginal bleeding

- Dyspnoea before exertion

- Dizziness

- Headache

- Chest pain

- Muscle weakness

- Calf pain or swelling (need to rule out thrombophlebitis)

- Preterm labour

- Decreased fetal movement

- Amniotic fluid leakage

Uterine activity has been measured in exercising pregnant women, ${ }^{78}$ and minimal or no changes were reported during the last eight weeks of pregnancy. In some reports, physical activity has been associated with an increase in uterine contractions.9 The magnitude of uterine contractions reported is usually low. There are only anecdotal reports that strenuous training may cause preterm labour. Nonetheless, until there is unequivocal evidence that strenuous exercise has no impact, a physically active woman with a history of, or who is at risk of, preterm labour should be advised to reduce her activity in the second and third trimesters. ${ }^{10}$

\section{NUTRITIONAL REQUIREMENTS}

After the 13th week of pregnancy, about 1.2 extra MJ (300 kcal) per day are required to meet the metabolic needs of pregnancy. ${ }^{11-13}$ This energy requirement is increased further when daily energy expenditure is increased through exercise. In weight bearing exercise, such as walking, the energy requirement progressively increases with the increase in weight during the course of the pregnancy. A related consideration to nutrition and exercise during pregnancy is adequate carbohydrate intake. Pregnant women use carbohydrates at a greater rate both at rest and during exercise than do non-pregnant women. ${ }^{15} 16$ It also appears that, during non-weight bearing exercise in pregnancy, there is preferential use of carbohydrates, possibly the result of the anaerobic component of this type of activity. ${ }^{17}$

CARDIOVASCULAR ADAPTATIONS Pregnancy induces profound alterations in maternal haemodynamics. Such changes include an increase in blood volume, heart rate, and stroke volume as well as cardiac output, and a decrease in systemic vascular resistance. ${ }^{13} 1819$ By midpregnancy, cardiac outputs are 30$50 \%$ greater than before pregnancy. ${ }^{20}$ Most studies show that maternal stroke volume increases by $10 \%$ by the end of the first trimester and is followed by a $20 \%$ increase in heart rate during the second and third trimesters. ${ }^{21}{ }^{22}$ Mean arterial pressure decreases $5-10 \mathrm{~mm} \mathrm{Hg}$ by the middle of the second trimester and then gradually increases back to prepregnancy levels. The decreased mean arterial pressure is the result of increased uterine vasculature, uteroplacental circulation, and the decrease in vascular resistance of predominantly the skin and kidney. ${ }^{21}$ These haemodynamic changes appear to establish a circulatory reserve necessary to provide nutrients and oxygen to both mother and fetus at rest and during moderate but not strenuous physical activity.

The cardiovascular changes associated with body posture is an important consideration for pregnant women both at rest and during exercise. After the first trimester, the supine position results in relative obstruction of venous return and therefore decreased cardiac output. For this reason, supine positions should be avoided as much as possible during rest and exercise. In addition, motionless standing is associated with a significant decrease in cardiac output, thus this position should be avoided. ${ }^{23}$ Conflicting evidence exists on maternal heart rate response to steady state submaximal exercise during pregnancy. ${ }^{24}$ Both blunted and normal responses to weight bearing and non-weight bearing exercise have been reported, ${ }^{24}{ }^{25}$ making use of heart rate monitoring to guide exercise intensity during pregnancy difficult.

\section{RESPIRATORY ADAPTATIONS}

Pregnancy is associated with profound respiratory changes: minute ventilation increases by almost $50 \%$, largely as a result of increased tidal volume. ${ }^{26}{ }^{27}$ This results in an increase in arterial oxygen tension to $106-108 \mathrm{~mm} \mathrm{Hg}$ in the first trimester, decreasing to a mean of 101-106 $\mathrm{mm} \mathrm{Hg}$ by the third trimester. ${ }^{28}$ There is an associated increase in oxygen uptake, and a $10-20 \%$ increase in baseline oxygen consumption. Physiological dead space during pregnancy remains unchanged..$^{26}{ }^{30}$ During treadmill exercise in pregnancy, arteriovenous oxygen difference is decreased. ${ }^{24}$ Because of the increased resting oxygen requirements and the increased work of breathing caused by pressure of the enlarged uterus on the diaphragm, there is decreased oxygen availability for the performance of aerobic exercise during pregnancy. Thus both subjective workload and maximum exercise performance are decreased. ${ }^{12}{ }^{27}$ However, in some fit women, there appear to be no associated changes in maximum aerobic power or acid-base balance during exercise in pregnancy compared with non-pregnant controls. ${ }^{13} 2931$

\section{THERMOREGULATORY CONTROL}

The cardiovascular system is affected the most by the increased metabolic demands of exercise, and therefore a major factor is the dissipation of the excess heat generated by exercise. During pregnancy, basal metabolic rate, and therefore heat production, is increased above non-pregnant levels. The increase in body temperature during exercise is directly related to the intensity of the exercise. During moderate intensity, aerobic exercise in thermoneutral conditions, the core temperature of nonpregnant women rises an average of $1.5^{\circ} \mathrm{C}$ during the first 30 minutes of exercise and then reaches a plateau if exercise is continued for an additional 30 minutes. ${ }^{16}$ A steady state of heat production versus heat dissipation is accomplished by increased conductance of heat from the core to the periphery through the cardiovascular system as well as through evaporative cooling through sweat. If heat production exceeds heat dissipation capacity, for example during exercise in hot, humid conditions or during very high intensity exercise, the core temperature will continue to rise. During prolonged exercise, loss of fluid as sweat may compromise heat dissipation. Maintenance of euhydration, and therefore blood volume, is critical to heat balance.

$$
\begin{aligned}
& \text { ". . an increase in maternal core } \\
& \text { temperature of more than } 1.5^{\circ} \mathrm{C} \\
& \text { during embryogenesis has been } \\
& \text { observed to cause major } \\
& \text { congenital malformations." }
\end{aligned}
$$

Data on the effects of exercise on core temperature during pregnancy are limited. ${ }^{12}{ }^{13} 16$ Fetal body core temperatures are about $1^{\circ} \mathrm{C}$ higher than maternal temperatures. In animal studies, an increase in maternal core temperature of more than $1.5^{\circ} \mathrm{C}$ during embryogenesis has been observed to cause major congenital malformations. ${ }^{32}$ These data coupled with the results of human studies suggest that hyperthermia in excess of $39^{\circ} \mathrm{C}$ during the first $45-60$ days of gestation may also be teratogenic in humans. ${ }^{32}{ }^{33}$ However, there have been no reports that hyperthermia associated with exercise is teratogenic in humans.

\section{FETAL RESPONSES TO MATERNAL EXERCISE}

In the past, the main concerns of exercise in pregnancy were focused on the fetus, and any potential maternal benefit was thought to be offset by potential risks to the fetus. In the uncomplicated pregnancy, fetal injuries are highly unlikely. Most of the potential fetal risks are hypothetical.

The principal question that remains to be answered is does the selective redistribution of blood flow during regular or prolonged exercise in pregnancy interfere with the transplacental transport of oxygen, carbon dioxide, and nutrients, 
and, if it does, what are the lasting effects, if any? The indirect evidence is that there are no lasting effects. Given this concern, water exercise may be an excellent choice of exercise during pregnancy because, during immersion, a centripetal shift in blood volume occurs.

It is well recognised that, during obstetric events, transient hypoxia could result initially in fetal tachycardia and an increase in fetal blood pressure. These fetal responses are protective mechanisms allowing the fetus to facilitate transfer of oxygen and decrease the carbon dioxide tension across the placenta. Any acute alterations could result in fetal heart rate changes, whereas chronic effects may result in intrauterine growth restriction. There are no reports to link such adverse events with maternal exercise.

Responses of fetal heart rate to maternal exercise have been the focus of numerous studies. ${ }^{27}$ 34-38 Most of the studies show a minimum or moderate increase in fetal heart rate by $10-30$ beats/min over baseline during or after maternal exercise. Fetal heart rate decelerations and bradycardia have been reported to occur with a frequency of $8.9 \%{ }^{36}$ The mechanism leading to fetal bradycardia during maternal exercise can only be speculated on: probably a vagal reflex, cord compression, or fetal head malposition. No associated lasting effects of the fetus have been reported.

Several studies ${ }^{89}{ }^{40}$ have attempted to assess umbilical blood flow during maternal exercise with Doppler velocimetry. These studies are technically difficult to conduct during exercise, so most measurements are taken before and after exercise, by which time any changes could have returned to normal.

Epidemiological studies have suggested for a long time that a link exists between strenuous physical activity, deficient diet, and the development of intrauterine growth restriction. This association appears to be particularly true for mothers engaged in physical work. It has also been reported that mothers whose occupation requires standing or repetitive, strenuous, physical work such as lifting have a tendency to deliver earlier and have small for gestational age infants. ${ }^{41-43}$ However, other reports have failed to confirm these associations, ${ }^{44}{ }^{45}$ suggesting that other factors or conditions, such as inefficient nutrition, have to be present for strenuous activities to affect fetal growth.

\section{"It appears that birth weight is not affected by exercise in women who have adequate energy intake."}

In another study it was concluded that mean birth weight is substantially lower when women exercised at or above $50 \%$ of preconception levels compared with non-exercisers. ${ }^{46}$ Another study $y^{47}$ found no difference between birth weight of offspring of vigorous exercisers and those of sedentary women, whereas others even found an increase in birth weight. ${ }^{48}$ It appears, however, that birth weight is not affected by exercise in women who have adequate energy intake. Reports on continuous physical training during pregnancy in athletes indicate that such activities carry very little risk. ${ }^{49}$

Although the reported birth weights are lower than expected by an average of $500 \mathrm{~g}$, these facts may be a partial explanation of some anecdotal reports of shorter duration of labour in some of these subjects.

The information available in the literature is too limited to allow risk assignment for either premature labour or fetal growth restriction in recreational or professional athlete exercising mothers, and the link to deficient diets has not been sufficiently addressed. Clinical observations indicate that patients at risk of premature labour may have labour triggered by exercise. Women who are diet conscious often do not receive the minimum required nutrients. The combined energy requirements of pregnancy and exercise coupled with poor weight gain may lead to fetal growth restriction.

\section{CLINICAL EVALUATION}

Exercise prescription requires knowledge of the potential risks and assessment of the physical ability to engage in various activities. Given the potential risks, albeit rare, thorough clinical evaluation of each pregnant woman should be conducted before an exercise programme is recommended. Routine prenatal care, as advocated in ACOG publications, is sufficient for monitoring the exercise programme.

\section{MEDICAL SCREENING BEFORE EXERCISE}

The overall health, obstetric, and medical risks should be reviewed before a pregnant woman is prescribed an exercise programme. In the absence of contraindications, a pregnant woman should be encouraged to engage in regular, moderate intensity physical activity to continue to derive the same associated health benefits during pregnancy as before pregnancy. However, there are contraindications to exercise because of preexisting or developing medical conditions, and pregnancy is not different. In addition, certain obstetric complications may develop in pregnant women regardless of the previous level of fitness, which could preclude them from continuing to exercise safely during pregnancy. The contraindications to exercise listed are suggested only as guides to determining the appropriateness of exercise during pregnancy for individual women. Box 3 highlights the warning signs of complications.

\section{EXERCISE PRESCRIPTION}

The elements of exercise prescription should be considered in every physical activity framework regardless of its purpose-that is, basic health, recreational pursuits, or competitive activities. Consideration should be given to the type and intensity of exercise as well as to the duration and frequency of exercise sessions to carefully balance between potential benefits and potential harmful effects. Additional attention should be given to progression in intensity over time.

\section{Basic exercise prescription for overall health and wellbeing Type of exercise}

Exercise prescription for the development and maintenance of fitness in non-pregnant women consists of activities to improve cardiorespiratory (aerobic exercise) and musculoskeletal (resistive exercise) status. $^{50}$ Exercise prescription in pregnancy should include the same elements. Aerobic exercise can consist of any activities that use large muscle groups in a continuous rhythmic manner-for example, activities such as walking, hiking, jogging/running, aerobic dance, swimming, cycling, rowing, cross country skiing, skating, dancing, and rope skipping. Because control of exercise intensity (see below) within rather precise limits is often desirable at the beginning of an exercise programme, the most easily quantified activities, such as walking or stationary cycling, are particularly useful. There are no data to support the restriction of pregnant women from participating in these activities, although some activities carry more risk than others. There are several activities that pose increased risks in pregnancy such as scuba diving and exertion in the supine position. Swimming, however, has not been associated with adverse effects and has the advantage of creating a buoyant condition that is well tolerated. Activities that increase the risk of falls, such as skiing, or those that may result in excessive joint stress, such as jogging and tennis, should include cautionary advice for most pregnant women, but evaluated on an individual basis with consideration for individual abilities. Certainly, the risk of related injuries is difficult to predict.

In addition to aerobic activities, activities that promote musculoskeletal fitness are part of an overall exercise prescription. Typically, these include both resistance training (weightlifting) and flexibility exercises. Limited information 
exists on strength training during pregnancy. In one study, ${ }^{51}$ individually prescribed strength training (one set of up to 12 repetitions) of multiple muscle groups was used as part of an overall conditioning programme for pregnant women. Fetal heart rates were monitored during training at 28 and 38 weeks gestation, and they remained unchanged. It was concluded that relatively low weights with multiple repetitions lifted through a dynamic range of motion appear to be a safe and effective type of resistance exercise during pregnancy. Although supporting data are lacking, it would be prudent to limit repetitive isometric or heavy resistance weightlifting and any exercises that result in a large pressor effect during pregnancy. Because of the increased relaxation of ligaments during pregnancy, flexibility exercise should be individualised for the same reason. Maintenance of normal joint range of motion, however, should not interfere with a moderate exercise routine in pregnant women. ${ }^{51}$

\section{Intensity of exercise}

Intensity is the most difficult component of an exercise regimen to prescribe for pregnant women. To derive health benefits, non-pregnant women are advised to participate in at least moderate intensity exercise. In the combined CDCACSM recommendations for physical activity and health, moderate exercise is defined as exercise of 3-4 METS or any activity that is equivalent in difficulty to brisk walking. ${ }^{4}$ There is no reason to alter this recommendation for pregnant women with no medical or obstetric complications. The recommended intensity of physical activity for developing and maintaining physical fitness is somewhat higher. The ACSM recommends that intensity should be $60-90 \%$ of maximal heart rate or $50-85 \%$ of either maximal oxygen uptake or heart rate reserve. The lower end of these ranges $(60-70 \%$ of maximal heart rate or $50-60 \%$ of maximal oxygen uptake) appears to be appropriate for most pregnant women who did not engage in regular exercise before pregnancy, and the upper part of these ranges should be considered for those who wish to continue to maintain fitness during pregnancy. In a meta-analysis study of exercise and pregnancy, it was reported that, with exercise intensities of $81 \%$ of heart rate maximum, no significant adverse effects were found. ${ }^{52}$

Given the variability in maternal heart rate responses to exercise, target heart rates cannot be used to monitor exercise intensity in pregnancy.

Ratings of perceived exertion have been found to be useful during pregnancy as an alternative to heart rate monitoring of exercise intensity. ${ }^{54}$ For moderate exercise, ratings of perceived exertion should be 12-14 (somewhat hard) on the 6-20 scale. Evidence of the efficacy of this approach is that, when exercise is self paced, most pregnant women will voluntarily reduce their exercise intensity as pregnancy progresses. ${ }^{55}$ Although an upper level of safe exercise intensity has not been established, women who were regular exercisers before pregnancy and who have uncomplicated, healthy pregnancies should be able to engage in high intensity exercise programmes, such as jogging and aerobics, with no adverse effects. The nutritional, cardiovascular, and musculoskeletal condition of the subject as well as fetal wellbeing should be periodically assessed during the prenatal office visits in pregnant women undertaking high intensity exercise programmes. Additional testing should be considered as clinically indicated-for example, non-stress fetal heart testing and ultrasound to assess fetal growth. Dietary modifications and changes in exercise routines should also be considered if clinically indicated.

\section{Duration of exercise}

Two concerns should be addressed before prescribing prolonged exercise (in excess of 45 minutes of continuous exercise) regimens for pregnant women. The first is thermoregulation. Exercise preferably should be performed in a thermoneutral environment or in controlled environmental conditions (air conditioning). Attention to proper hydration and subjective feelings of heat stress are essential. The second concern is energy balance. Energy costs of fitness exercise should be estimated and balanced by appropriate energy intakes. Setting of limits to exercise durations is not possible because of the reciprocal relation between exercise intensity and duration. It should be noted that, in studies in which exercise was self paced, in a controlled environment, core temperatures rose less than $1.5^{\circ} \mathrm{C}$ over 30 minutes and stayed within safe limits. ${ }^{10}$ Accumulating the activity in shorter exercise periods, such as 15 minute periods, may obviate concerns related to thermoregulation and energy balance during exercise sessions. ACSM recommends that non-pregnant women exercising to increase or maintain fitness may exercise for up to 60 minutes per exercise session. ${ }^{52}$

\section{Frequency of exercise}

In the current CDC-ACSM recommendations for exercise aimed at health and wellbeing, the recommendation for nonpregnant women is that an accumulation of 30 minutes a day of exercise occur on most if not all days of the week. In the absence of either medical or obstetric complications, pregnant women could adopt the same recommendation.

\section{Progression}

Pregnant women who have been sedentary before pregnancy should follow a gradual progression of up to 30 minutes a day. This recommendation is not different from that for non-pregnant sedentary women who begin an exercise programme. Pregnancy is not a time for greatly improving physical fitness. Therefore, women who have attained a high level of fitness through regular exercise before pregnancy should exercise caution in engaging in higher levels of fitness activities during pregnancy. Further, they should expect overall activity and fitness levels to decline somewhat as pregnancy progresses. ${ }^{55}$

\section{Recreational activities}

Most reports of participation in active recreational activities during pregnancy are anecdotal in nature. In general, participation in a wide range of recreational activities appears to be safe. The safety of each sport is largely determined by the specific movements required by that sport. Activities with a high risk of falling or those with a high risk of abdominal trauma should be considered undesirable. ${ }^{56}$ Participation in recreational sports with a high potential for contact, such as ice hockey, soccer, and basketball, could result in serious trauma to both mother and fetus. Similarly, recreational activities with increased risk of falling, such as gymnastics, horseback riding, downhill skiing, and vigorous racquet sports, have inherent high risk of trauma in pregnant and non-pregnant women. Scuba diving should be avoided throughout pregnancy because the fetus is at increased risk of decompression sickness secondary to the inability of the fetal pulmonary circulation to filter bubble formation. ${ }^{57}$ As for exertion at altitude, reports are available for activities at less than $2500 \mathrm{~m}$ (6000 feet). In one study conducted at $2500 \mathrm{~m}$, it was concluded that pregnant women may engage in periods of exercise and/or moderate physical tasks, but are limited in performing high intensity physical activities. No adverse fetal responses were recorded during this study. ${ }^{58}$ Other studies confirm the lack of adverse effects on the fetus at altitudes typically used for mountain sports such as hiking or skiing (less than $2500 \mathrm{~m}) .^{59}$ All women who are recreationally active should be aware of signs of altitude sickness for which they should stop exercise, descend from altitude, and seek medical attention (box 3 ).

\section{Water exercise}

The major effect of immersion is a redistribution of extravascular fluid into vascular space, resulting in an increase in blood volume..$^{60}{ }^{61}$ This effect occurs very rapidly and is proportional to the depth of immersion, leading to a decrease in 
systemic blood pressure (both systolic and diastolic). These changes are accompanied by a decrease in antidiuretic hormone, aldosterone, and plasma renin activity while the atrial natriuretic factor decreases.

The shift in blood volume leads to ventilatory changes with a decline in vital capacity, ventilation capacity, and expiratory reserve volume. ${ }^{62}$ Immersion is ideal for dissipating exercise induced increased temperature during exercise in pregnancy. ${ }^{55}$

$$
\begin{aligned}
& \text { "No adverse effects on the fetus } \\
& \text { have been reported to occur } \\
& \text { during water exercise in } \\
& \text { pregnancy." }
\end{aligned}
$$

In longitudinal studies of immersion exercise in pregnancy at $60 \%$ maximal oxygen consumption, it was found to be a safe activity, with advantageous effects on oedema, thermal regulation, and buoyancy, thus minimising the risk of joint injuries. ${ }^{63}$ Furthermore, no adverse effects on the fetus have been reported to occur during water exercise in pregnancy.

\section{COMPETITIVE ATHLETICS}

Competitive athletes are likely to encounter the same limitations as faced by recreational athletes during pregnancy. The competitors tend to maintain a more strenuous training schedule throughout pregnancy and to resume high intensity postpartum training sooner. The concerns of the pregnant, competitive athlete fall into two general categories: $(a)$ the effects of pregnancy on competitive ability; $(b)$ the effects of strenuous training and competition on pregnancy, particularly the fetus. Such athletes would certainly require closer obstetric supervision than the routine prenatal care. Additional testing and intervention should occur as clinically indicated.

As pregnancy progresses, several changes occur that will prevent the athlete from attaining the same performance levels as before pregnancy. Weight gain, by itself and in the presence of laxity of joints and ligaments and change in the centre of gravity, will cause unavoidable limitations in most sporting activities. The ability to stop and start or to change direction will progressively decrease. Any attempts to substitute compensatory movements for finely tuned skill movements result in inefficient movement, decrease in competitive ability, and increase in the risk of injury. Performance in sports in which endurance is important may be adversely affected by the physiological anaemia commonly associated with the increased blood volume of pregnancy.

Despite the fact that pregnancy adversely affects performance in the competitive athlete, most elite athletes prefer to continue to train during pregnancy. The relatively high intensity, long duration, and frequent workout schedules of most competitive athletes may place them at greater risk of thermoregulatory complications during pregnancy. ${ }^{10}$ Particular attention should be paid to maintaining proper hydration during and between these exercise sessions. Fluid balance during an exercise session can be monitored by weighing before and after the session. Any loss of weight is fluid loss that should be made up before the next exercise session ( $1 \mathrm{lb}$ weight loss $\approx 1$ pint of fluid).

Because of the type (high intensity, prolonged, and frequent) of training done by elite athletes, it is likely that weight gain will be less for both mother and fetus than for sedentary women. This lower birth weight has been attributed to decreased neonatal fat mass. ${ }^{46}$

\section{SPECIAL POPULATIONS}

Pregnant women with diabetes, morbid obesity, or chronic hypertension should have individualised exercise prescription. The information available in the literature is limited with regard to the role of physical activity for these women. Two randomised trials of exercise training in women with gestational diabetes have been published. ${ }^{64-66}$ In one study, arm ergometry exercise three times a week for about 20 minutes a session at 50\% maximal oxygen consumption resulted in normalisation of glycaemic control after four weeks in contrast with diet alone. ${ }^{67}$ A second study included 41 women at 28-33 weeks gestation who, despite dietary treatment, had persistent fasting hyperglycaemia of $105-140 \mathrm{mg} /$ dl. Study control subjects were treated with insulin. The exercise patients performed moderate cycle exercise three times a week and maintained an active lifestyle for the duration of pregnancy. Through this regimen, the exercising patients maintained euglycaemia and did not require insulin. In a study of women with type I diabetes mellitus, a postprandial walking programme did not achieve the desirable glycaemic control. ${ }^{68}$ Epidemiological data suggest that exercise may even be beneficial in the primary prevention of gestational diabetes particularly in morbidly obese women (body mass index $>33$ ), but not in women of normal weight. ${ }^{69}$ The American Diabetes Association has endorsed exercise as "a helpful adjunctive therapy" for gestational diabetes when euglycaemia is not achieved by diet alone. ${ }^{70}$ There is currently no information available on the effect of exercise on women with chronic hypertension. The standard of care for women with pregnancy induced hypertension is to limit physical activity.

\section{EXERCISE IN THE POSTPARTUM} PERIOD

Many of the physiological and morphological changes of pregnancy persist for four to six weeks post partum. Thus, exercise routines may be resumed only gradually after pregnancy and should be individualised. Physical activity can thus be resumed as soon as physically and medically safe. This will certainly vary from one woman to another, with some being capable of engaging in an exercise routine within days of delivery. There are no published studies to indicate that, in the absence of medical complications, rapid resumption of activities will result in adverse effects. Undoubtedly, having undergone detraining, resumption of activities should be gradual. No known maternal complications are associated with resumption of training. ${ }^{10}$ Moderate weight reduction while nursing is safe and does not compromise neonatal weight gain. ${ }^{71}$ Failure to gain weight is associated with decreased milk production, which may be secondary to inadequate fluid or nutritional intake to balance training induced outputs. Nursing women should consider feeding their infants before exercising in order to avoid the discomfort of engorged breasts. $^{72}{ }^{73}$ In addition, nursing before exercise avoids the potential problems associated with increased acidity of milk secondary to any build up of lactic acid. Finally, a return to physical activity after pregnancy has been associated with decreased postpartum depression, but only if the exercise is stress relieving and not stress provoking. ${ }^{74}$

\section{SUMMARY}

Pregnancy should not be a state of confinement, and pregnant women with uncomplicated pregnancies should be encouraged to continue and engage in physical activities. Recreational and competitive athletes with uncomplicated pregnancies may remain active during pregnancy, and modify their usual exercise routines as indicated in this review. All active pregnant women should be examined periodically to assess the effects of their exercise programmes on the developing fetus, so that adjustments can be made if necessary. Women with medical or obstetric complications should be carefully evaluated before recommendations on physical activity participation during pregnancy are made. Despite the fact that pregnancy is associated with profound anatomical and physiological changes, exercise has minimal risks and confirmed benefits for most women.

Br J Sports Med 2003;37:6-12

\section{Authors' affiliations}

R Artal, Saint Louis University, St Louis, MO, USA 
Correspondence to: Dr Artal, 6420 Clayton Road, Ste 290 St Louis, MO 63117, USA; artalr@slucare 1.sluh.edu

\section{REFERENCES}

1 ACOG Committee. Opinion no. 267

exercise during pregnancy and the postpartum period. Obstet Gynecol 2002;99:171-3.

2 Blair SN, Kohl HW, Gordon NF. How much physical activity is good for health? Annu Rev Publ Health 1992:13:99-126.

3 Blair SN. Physical activity, fitness, and coronary heart disease. In: C Bouchard, RI Shephard, T Stephens, eds. Physical activity, fitness, and health: international proceedings and consensus statement. Champaign, IL: Human Kinetics, 1994:591-608.

4 Pate RR, Pratt M, Blair SN, et al. A recommendation from the Centers for Disease Control and Prevention and the American College of Sports Medicine. JAMA 1995;273:402-7.

5 Karzel RP, Friedman M. Orthopedic injuries in pregnancy. In: Artal R, Wiswell RA, Drinkwater BL, eds. Exercise in pregnancy. 2nd ed. Baltimore: Williams and Wilkins, 1991.

6 Calguneri M, Bird HA, Wright V. Changes in joint laxity occurring during pregnancy. Ann Rheum Dis 1982;41:126-8

7 Artal R, Platt LD, Sperling M, et al. Exercise in pregnancy. I. Maternal cardiovascular and metabolic response in normal pregnancy. $A m$ J Obstet Gynecol 1981;140:123-7.

8 Veille J-C, Hohimer RA, Burry K, et al. The effect of exercise on uterine activity in the last eight weeks of pregnancy. Am J Obstet Gynecol 1985;151:727-30

9 Grisso JA, Main DM, Chiu G, et al. Effects of physical activity and life-style factors on uterine contraction frequency. Am J Perinatol 1992:9:489-92

10 Hale RW, Milne L. The elite athlete and exercise in pregnancy. Semin Perinatol 1996;20:277-84.

11 NRC (National Research Council) Recommended dietary allowances. 10th ed. Washington, DC: National Academy of Sciences, 1989.

12 Clapp JF III. Exercise in pregnancy: a brief clinical review. Fetal Medical Review 1990;161:1464-9.

13 Artal R, Wiswell RA, Drinkwater BL, eds. Exercise in pregnancy. 2nd ed. Baltimore: Williams and Wilkins, 1991

14 Butterfield G, King JC. Nutritional needs of physically active pregnant women. In: Artal $R$, Wiswell RA, Drinkwater BL, eds. Exercise in pregnancy. 2nd ed. Baltimore: Williams and Wilkins, 1991

15 Clapp JF III, Seaward BL, Sleamaker RH, et al. Maternal physiologic adaptations to early human pregnancy. Am J Obstet Gynecol 1988;159:1456-60.

16 Soultanakis HN, Artal R, Wiswell RA. Prolonged exercise in pregnancy: glucose homeostasis, ventilatory and cardiovascula responses. Semin Perinatol 1996;20:315-27.

17 Artal R, Masaki DI, Khodiguian N, et al. Exercise prescription in pregnancy: weight-bearing versus non-weight-bearing exercise. Am J Obstet Gynecol 1989;161:1464-9.

18 Clark SL, Cotton DB, Lee W, et al. Central hemodynamic assessment of normal term pregnancy. Am J Obstet Gynecol 1989; 161:1439-42.

19 Wolfe LA, Ohtake PJ, Mottola MF, et al. Physiological interactions between pregnancy and aerobic exercise. Exerc Sport Sci Rev 1989; 17:295-351.

20 Morton MJ. Maternal hemodynamics in pregnancy. In: Artal R, Wiswell RA Drinkwater BL, eds. Exercise in Pregnancy. 2nd ed. Baltimore: Williams and Wilkins, 1991.

21 Pivaranik JM. Cardiovascular responses to aerobic exercise during pregnancy and postpartum. Semin Perinatol 1996;20:242-9.

22 Morton JM, Paul MS, Campos GR, et al. Exercise dynamics in late gestation. Am $J$ Obstet Gynecol 1985;152:91-7.
23 Clark SL, Cotton DB, Pivarnik JM et al. Position change and central hemodynamic profile during normal third-trimester pregnancy and post partum. Am J Obstet Gynecol $1991 ; 164: 883-7$

24 Pivarnik JM, Lee W, Clark SL, et al. Cardiac output responses of primigravid women during exercise determined by the direct Fick technique. Obstet Gynecol 1990;75:954-9

25 McMurray RG, Hackney AC, Katz VL, et al. Pregnancy-induced changes in the maximal physiological responses during swimming. $J$ Appl Physiol 1991;71:1454-9.

26 Prowse CM, Gaensler EA. Respiratory and acid-base changes during pregnancy. Anesthesiology 1965;26:381-92.

27 Artal R, Wiswell R, Romen Y, et al. Pulmonary responses to exercise in pregnancy. Am J Obstet Gynecol 1986;154:378-83.

28 Templeton A, Kelman GR. Maternal blood-gases $\left(\mathrm{PAO}_{2}-\mathrm{PaO}_{2}\right)$ physiological shunt and VD/VT in normal pregnancy. $\mathrm{Br}$ Anesthesiol 1976;48:1001-4.

29 Pivarnik JM, Lee W, Spillman T, et al. Maternal respiration and blood gases during aerobic exercise performed at moderate altitude. Med Sci Sports Exerc 1992;24:868-72

30 Sady SP, Carpenter MW, Thompson PD, et al. Cardiovascular response to cycle during and after pregnancy. J Appl Physiol 1989:66:336-41.

31 Lotgering FK, Van Doorn MB, Struijk PC, et al. Maximal aerobic exercise in pregnant women: heart rate, $\mathrm{O}_{2}$ consumption, $\mathrm{CO}_{2}$ production and ventilation. J Appl Physiol 1991;70:1016-23.

32 Milunsky A, Ulcickas M, Rothman KJ, et al. Maternal heat exposure and neural tube defects. JAMA 1992;268:882-5.

33 Edwards MJ. Hyperthermia as a teratogen: a review of experimental studies and their clinical significance. Teratog Carcinog Mutagen 1986:6:563-82

34 Collings CMS, Curet LB, Mullin JP. Materna and fetal responses to a maternal aerobic exercise program. Am J Obstet Gynecol 1983;145:702-7

35 Clapp JF 3rd. Fetal heart rate responses to running in midpregnancy and late pregnancy. Am J Obstet Gynecol 1985;153:251-2.

36 Artal R. Exercise and diabetes mellitus. A brief review. Sports Med 1990;9:261-5.

37 Carpenter MW, Sady SP, Hoegsberg B, et al. Fetal heart rate response to maternal exertion. JAMA 1988;259:3006-9.

38 Wolfe LA, Lowe-Wylde SJ, Tranmer JE, et al. Fetal heart rate during maternal static exercise [abstract]. Can J Sport Sci 1988;13:95P-6P.

39 Erkkola RU, Pirhonen JP, Kiwijaroi AK. Flow velocity waveforms in uterine and umbilical arteries during submaximal bicycle exercise in normal pregnancy. Obstet Gynecol 1992;79:611-15.

40 Morrow RJ, Ritchie JWK, Bull SB. Fetal and maternal hemodynamic response to exercise in pregnancy assessed by Doppler ultrasound. Am J Obstet Gynecol 1989;160:138-40.

41 Naeye RL, Peters E. Working during pregnancy, effects on the fetus. Pediatrics 1982;69:724-7.

42 Launer LH, Villar J, Kestler E, et al. The effect of maternal work on fetal growth and duration of pregnancy: a prospective study. Br J Obstet Gynaecol 1990:97;62-70.

43 McDonald AD, McDonald JC, Armstrong B, et al. Prematurity and work in pregnancy. $\mathrm{Br} J$ Ind Med 1988;45:56-62.

44 Saurel-Cubizolles MJ, Kaminski M. Pregnant women's working conditions and their changes during pregnancy: a national study in France. $\mathrm{Br} J$ Ind Med 1987;44:236-43.

45 Ahlborg G, Bodin L, Hogstedt C. Heavy lifting during pregnancy: a hazard to the fetus? A prospective study. Int J Epidemiol 1990;19:90-7

46 Clapp JF, Capeless EL. Neonata morphometrics after endurance exercise during pregnancy. Am J Obstet Gynecol 1990;163:1805-11.
47 Sternfeld B, Quesenberry CP Jr, Eskenazi B, et al. Exercise during pregnancy and pregnancy outcome. Med Sci Sports Exerc 1995;27:634-40.

48 Hatch MC, Shu X-O, Mclean DE, et al. Maternal exercise during pregnancy, physical fitness and fetal growth. Am J Epidemiol 1993; 137: 1 105-14

49 Erdelyi GJ. Gynecological survey of female athletes. J Sports Med Phys Fitness 1962;2:174-5

50 Pollock ML, Gaesser GA, Butcher JD. The recommended quantity and quality of exercise for developing and maintaining cardiorespiratory and muscular fitness, and flexibility in healthy adults. Med Sci Sports Exerc 1998:30:975-91.

51 Hall DC, Kaufmann DA. Effects of aerobic and strength conditioning on pregnancy outcomes. Am J Obstet Gynecol 1987;157: 1 199-203.

52 ACSM. Guidelines for exercise testing and prescription. 6th ed. Philadelphia: Lippincott Williams \& Wilkins, 2000

53 Lokey EA, Tran ZV, Wells CL, et al. Effects of physical exercise on pregnancy outcomes: a meta-analytic review. Med Sci Sports Exerc 1991;23:1234-9.

54 Pivarnik JM. Maternal exercise during pregnancy. Sports Med 1994;18:215-17.

55 McMurray RG, Mottola MF, Wolfe LA, et al. Recent advances in understanding maternal and fetal responses to exercise. Med Sci Sports Exerc 1993;25:1305-21

56 Artal R. Exercise during pregnancy. Safe and beneficial for most. Physician and Sports Medicine 1999;27:51-60.

57 Camporesi EM. Diving and pregnancy. In Artal R, ed. Semin Perinatol 1996;20:292-302

58 Artal R, Fortunato V, Welton A, et al. A comparison of cardiopulmonary adaptations to exercise in pregnancy at sea level and altitude. Am J Obstet Gynecol 1995; 172:1170-80.

59 Huch R. Physical activity at altitude in pregnancy. In: Artal R, ed. Semin Perinatol 1996;20:303-14.

60 Epstien M. Water immersion and the kidney: implications for volume regulation Undersea. Biomed Res 1984;II: 1 14-37.

61 Epstein M, Loutzenhiser R, Friedland E, et al Relationship of increased plasma atrial natriuretic factor renal sodium handling during immersion-induced central hypervolemia in normal humans. J Clin Invest 1987:79:738-45

62 Berry MJ, McMurray RG, Katz VL. Pulmonary and ventilatory responses to pregnancy, immersion and exercise. J Appl Physiol 1989;66:857-62

63 Katz VL, McMurray R, Berry M, et al. Fetal and uterine responses to immersion and exercise. Obstet Gynecol 1988;72:225-30.

64 Jovanovic-Peterson L, Peterson CM. Exercise and the nutritional management of diabetes during pregnancy. Obstet Gynecol Clin North Am 1996;23:75-86

65 Bung P, Artal R, Khodigmian N, et al. Exercise in gestational diabetes: an optional therapeutic approach? Diabetes 1991;40:182-5.

66 Bung P, Artal R. Gestational diabetes and exercise. Semin Perinatol 1996:20;328-33.

67 Jovanovic-Peterson L, Durak EP, Peterson CM. Randomized trial of diet versus diet plus cardiovascular conditioning on glucose levels in gestational diabetes. Am J Obstet Gynecol 1989;161:415-19.

68 Hollingsworth DR, Moore TR. Postprandial walking exercise in pregnant insulin-dependent (type I) diabetic women: Reduction of plasma lipid levels but absence of a significant effect on glycemic control. Am J Obstet Gynecol 1987; 157:1359-63.

69 Dye TD, Knox KL, Artal R, et al. Physical activity, obesity, and diabetes in pregnancy. Am J Epidemiol 1997; 146:961-5.

70 Jovanovic L. American Diabetes Association's Fourth International Workshop: conference on gestational diabetes mellitus: summary and discussion. Therapeutic interventions. 1998;21(suppl 2);B131-7. 
71 McCrory MA, Nommsen-Rivers LA, Mole PA et al. Randomized trial of short-term effects of dieting compared with dieting plus aerobic exercise on lactation performance. Am J Clin Nutr 1999:69:959-67.

72 Kulpa PJ, White BM, Visscher R. Aerobic exercise in pregnancy. Am J Obstet Gynecol 1987; 156:1395-403.

73 Kulpa P. Exercise during pregnancy and post partum. In: Agostini R, ed. Medical and orthopedic issues of active and athletic women. Philadelphia: Hanley and Belfus, 1994:191-9.

74 Koltyn KF, Schultes SS. Psychological effects of an aerobic exercise session and a rest session following pregnancy. J Sports Med Phys Fitness 1997;37:287-91.

\section{COMMENTARY}

n March 2002, Sports Medicine Australia (SMA) also released a consensus statement on exercise in pregnancy, independently of the American College of Obstetricians and Gynecologists (ACOG). Not surprisingly most of the recommendations are similar, with an emphasis on encouraging activity during pregnancy because of its short and long term benefits. The latest guidelines also acknowledge that an exercise programme can be started or increased in a normal, healthy pregnancy.

After extensive review of the literature, both guidelines concede that there are no reported adverse pregnancy outcomes related to exercise during pregnancy and most of the potential risks such as reduced transplacental oxygen and nutrients, and hypothermia related teratogenesis are hypothetical. The ACOG (and SMA) no longer recommend heart rate targets to assess intensity of exercise but prefer self regulation and scales of perceived exertion. The ACOG guidelines only briefly comment on contact sports, advising that there may be a risk of trauma and therefore they should be avoided.

SMA's statement was initiated after a ban was placed on pregnant women participating in the moderate contact sport of netball. These guidelines therefore describe in more detail related research into abdominal trauma and sports injuries. This discussion suggests that many contact sports may in fact pose no serious risk to the mother or fetus.

The ACOG's guidelines for the first time provide helpful comment on competitive athletes and special groups (diabetic, obese, and hypertensive) of pregnant women. Overall, the latest ACOG guidelines are more comprehensive and generous in their advice to pregnant women. Like SMA, they encourage women to exercise before, during, and after pregnancy after appropriate medical assessment and advice.

S White

Olympic Park Sports Medicine Centre, Swan Street, Melbourne, Victoria 3000, Australia; susanwhite@optusnet.com.au

1 Sports Medicine Australia. SMA statement: the benefits and risks of exercise during pregnancy. J Sci Med Sport 2002;5:11-19. 